\title{
Effects of Patient Characteristics on Diagnostic Performance of Self-Collected Samples for SARS-CoV-2 Testing
}

Sarah E. Smith-Jeffcoat, Mitsuki Koh, Adam Hoffman, Paulina A. Rebolledo, Marcos C. Schechter, Halie K. Miller, Sadia Sleweon, Rebecca Rossetti, Vyjayanti Kasinathan, Talya Shragai, Kevin O'Laughlin, Catherine C. Espinosa, George M. Khalil, AdeSubomi O. Adeyemo, Anne Moorman, Brenda L. Bauman, Kahaliah Joseph, Michelle O'Hegarty, Nazia Kamal, Hany Atallah, Brooks L. Moore, Caitlin D. Bohannon, Bettina Bankamp, Claire Hartloge, Michael D. Bowen, Ashley Paulick, Amy S. Gargis, Christopher Elkins, Rebekah J. Stewart, Juliana da Silva, Caitlin Biedron, Jacqueline E. Tate, Yun F. Wang, Hannah L. Kirking, the CDC COVID-19 Response Team ${ }^{1}$

\begin{abstract}
We evaluated the performance of self-collected anterior nasal swab (ANS) and saliva samples compared with healthcare worker-collected nasopharyngeal swab specimens used to test for severe acute respiratory syndrome coronavirus 2 (SARS-CoV-2). We used the same PCR diagnostic panel to test all self-collected and healthcare worker-collected samples from participants at a public hospital in Atlanta, Georgia, USA. Among 1,076 participants, $51.9 \%$ were men, $57.1 \%$ were $\geq 50$ years of age, $81.2 \%$ were Black (non-Hispanic), and $74.9 \%$ reported $\geq 1$ chronic medical condition. In total, $8.0 \%$ tested positive for SARS-CoV-2. Compared with nasopharyngeal swab samples, ANS samples had a sensitivity of $59 \%$ and saliva samples a sensitivity of $68 \%$. Among participants tested 3-7 days after symptom onset, ANS samples had a sensitivity of $80 \%$ and saliva samples a sensitivity of $85 \%$. Sensitivity varied by specimen type and patient characteristics. These findings can help physicians interpret PCR results for SARS-CoV-2.
\end{abstract}

Author affiliations: Centers for Disease Control and Prevention, Atlanta, Georgia, USA (S.E. Smith-Jeffcoat, M. Koh, H.K. Miller, S. Sleweon, R. Rossetti, T. Shragai, K. O'Laughlin, C.C. Espinosa, G.M. Khalil, A.O. Adeyemo, A. Moorman, B.L. Bauman, K. Joseph, M. O'Hegarty, N. Kamal, C.D. Bohannon, B. Bankamp, C. Hartlodge, M.D. Bowen, A. Paulick, A.S. Gargis, C. Elkins, R.J. Stewart, J. da Silva, C. Biedron, J.E. Tate, H.L. Kirking); Public Health Institute-CDC Global Health Fellowship Program, Atlanta (M. Koh); Emory University, Atlanta (A. Hoffman, P.A. Rebolledo, M.C. Schechter, V. Kasinathan, H. Atallah, B.L. Moore, Y.F. Wang); Grady Memorial Hospital, Atlanta (A. Hoffman, P.A. Rebolledo, M.C. Schechter, V. Kasinathan, H. Atallah, B.L. Moore, Y.F. Wang)

DOI: https://doi.org/10.3201/eid2708.210667
Detection of severe acute respiratory syndrome coronavirus 2 (SARS-CoV-2), the virus that causes coronavirus disease (COVID-19), originally relied mainly on nasopharyngeal swab (NPS) samples collected by healthcare workers (HCWs). However, NPS sample collection requires substantial amounts of time and personal protective equipment (PPE) that could be preferentially used for patient care. In light of $>98$ million confirmed COVID-19 cases globally as of January 27, 2021, relying solely on HCW-collected specimens for testing is not feasible (1). During the COVID-19 pandemic, many healthcare sites have experienced shortages of PPE and testing supplies. In addition, NPS sample collection often causes coughing or sneezing, which can generate infectious aerosols and thereby put the HCW at increased risk for exposure (2). Furthermore, NPS collection can cause discomfort and occasional nosebleeds, possibly affecting a patient's willingness to be retested. The use of self-collected saliva and anterior nasal swab (ANS) samples reduces HCW contact, limits need for PPE, and preserves transport media and other collection supplies needed for NPS samples.

Various upper respiratory specimen types, including saliva and oral swab samples, have demonstrated similar sensitivity to NPS samples in nucleic acid amplification tests for SARS-CoV-2 (3-6). However, most patients in these studies reported the recent onset of respiratory symptoms. Other investigations have shown that many infected persons, especially those who are young and otherwise healthy, are

${ }^{1}$ Members are listed at the end of this article. 
asymptomatic or have mild symptoms (7-9). SARSCoV-2 RNA has been detected in NPS samples nearly 2 months after initial detection; however, the performance of self-collected ANS and saliva samples of patients with prolonged viral shedding remains unclear $(10,11)$. Understanding how these less invasive, selfcollected specimens perform in a variety of contexts can inform testing strategies. We compared the diagnostic performance of self-collected ANS and saliva samples and HCW-collected NPS samples used in SARS-CoV-2-specific PCR by patient characteristics and symptom status.

\section{Methods}

We recruited patients from several inpatient and outpatient departments of Grady Memorial Hospital (Atlanta, GA, USA), where a high proportion of patients are uninsured $(24 \%)$ or have Medicare/Medicaid insurance $(57 \%)$ (12). Patients were eligible if their treating physician ordered collection of an NPS sample for SARS-CoV-2-specific reverse transcription PCR (RT-PCR) for any reason, including diagnostic (e.g., patients were symptomatic or exposed) or screening (e.g., preoperative requirement or before admission for non-COVID-19 reasons) purposes. Patients were excluded if they were unable to provide consent, declined consent, were $<18$ years of age, had a contraindicated NPS specimen (e.g., had a condition that prevented NPS sample collection), were unable to self-collect specimens, or had previously participated in this investigation. Trained interviewers used a standardized questionnaire to collect data on patient demographics, reason for visit, current and previous symptoms, and medical history (including previous SARS-CoV-2 testing). Each participant received a US \$25 gift card.

During interviews, patients were given an infographic outlining steps for self-collection of saliva and ANS samples (Appendix Figure, https:/ / wwwnc.cdc. gov/EID/article/27/8/21-0667-App1.pdf) (13). Patients self-collected raw (unenhanced) saliva in a 50$\mathrm{mL}$ tube. Patients then inserted a miniature flockedtip swab into 1 anterior naris, twirled the swab for 10 seconds, removed the swab and placed it directly into the other naris, and twirled it again for 10 seconds. Patients inserted the swab into $3 \mathrm{~mL}$ of viral transport media. After the interview and self-collection of specimens, a HCW collected an NPS sample from the participant and inserted the swab into $3 \mathrm{~mL}$ of viral transport media. Hospital laboratory staff conducted RT-PCR on the NPS sample on the same day; these results were used to inform clinical care and were not included in the performance analysis. NPS samples then were aliquoted and transferred to the Centers for Disease Control and Prevention (CDC) for RT-PCR. CDC staff extracted nucleic acid and tested samples using the CDC 2019-nCoV Real-Time Reverse Transcription PCR (rRT-PCR) Diagnostic Panel, which is selective for the SARS-CoV-2 nucleocapsid 1 (N1) and 2 (N2) genes, as per the Emergency Use Authorization Instructions for Use $(14,15)$ (Appendix).

We entered and stored completed questionnaires and laboratory results in a REDCap version 10.0.8 (https://www.project-redcap.org) database hosted at CDC. We grouped patients according to COVID-19 symptom status: always asymptomatic participants reported no COVID-19 symptoms at specimen collection or in the previous 14 days; currently asymptomatic participants reported no COVID-19 symptoms at specimen collection but had symptoms in the previous 14 days; and currently symptomatic participants reported COVID-19 symptoms at specimen collection. We categorized symptoms according to previously defined case definitions (16-18) (Appendix). We calculated sample size using a 1-sided, 1-sample proportions test with a continuity correction to determine whether sensitivity of self-collected samples was $\geq 90 \%$ compared with HCW-collected NPS samples, assuming that NPS samples had a true sensitivity of $98 \%$ (3). Using a $=0.05,80 \%$ power, and $5 \%$ NPS percent positivity, we calculated the minimum sample size to be 920 and the required number of positive self-collected specimens to be 46 .

We compiled demographic and clinical characteristics for patients according to the results of their NPS samples. To analyze the benefit of using both self-collected ANS and saliva specimens for diagnosis, we merged each patient's ANS and swab sample results to create a self-collected combination result. If $\geq 1$ self-collected specimen was positive, we marked that patient's self-collected combination result as positive. If neither was positive and $\geq 1$ was negative, then we marked that patient's self-collected combination result as negative. We calculated sensitivity, specificity, positive predictive value (PPV), and negative predictive value (NPV) of ANS, saliva, and self-collected combination samples compared with NPS samples for all patients who had a definitive (i.e., positive or negative) NPS result and $\geq 1$ self-collected specimen. Because NPS samples do not show all SARS-CoV-2 infections, we reran the sensitivity analysis with a combined variable for any positive result from ANS, saliva, or NPS samples as the comparator. We compiled proportions of concordant and discordant results for each self-collected and HCW-collected sample and calculated Cohen's K 
coefficient to compare result agreement by specimen type. We calculated the sensitivity of self-collected specimens by patient characteristics and determined significant differences using a 1-sample, 2-sided test of proportions $(\mathrm{p}<0.05)$. We used the Pearson correlation coefficient to compare the cycle threshold $\left(C_{t}\right)$ values of positive self-collected and HCWcollected specimens; we used the Mann-Whitney $U$ test to compare the $C_{t}$ values of NPS samples by patient characteristic. We analyzed the data in $\mathrm{R}$ version 4.0.2 (The R Project for Statistical Computing, https://www.r-project.org).

This investigation was reviewed by CDC and conducted in accordance with applicable federal law and CDC policy (e.g., 45 C.F.R. part 46, 21 C.F.R. part 56, 42 U.S.C.; 241(d); 5 U.S.C. 552a; 44 U.S.C. 3501 et seq.). This investigation was determined to be an exempt public health activity by the Emory University Institutional Review Board and Grady Memorial Hospital Research Oversight Committee.

\section{Results}

During August 31-November 23, 2020, a total of 1,096 patients consented to and enrolled in the study; 20 were excluded because they did not meet inclusion criteria (Figure 1). Among 1,076 participants, overall positivity of any specimen was $8.0 \%$; NPS samples had $7.4 \%$ positivity, ANS samples had $4.4 \%$ positivity, and saliva samples had $4.8 \%$ positivity. Among the 1,076 participants, 51.9\% (559) were men, $57.1 \%$ (614) were $\geq 50$ years of age, $81.2 \%$ (874) were Black (non-Hispanic), and 74.9\% (806) had $\geq 1$ chronic medical condition (Table 1$)$. Most $(80.0 \%$; 861) participants were enrolled in the emergency department: nearly half sought care for a COVID-19related concern $(18.2 \% ; 196)$ or had a chief complaint including COVID-19-like symptoms (30.6\%; 329). Over half $(56.7 \% ; 610)$ of participants had $\geq 1$ current COVID-19 symptom; among currently symptomatic participants, $68.9 \%$ (420) reported symptom onset $\leq 1$ week previously.

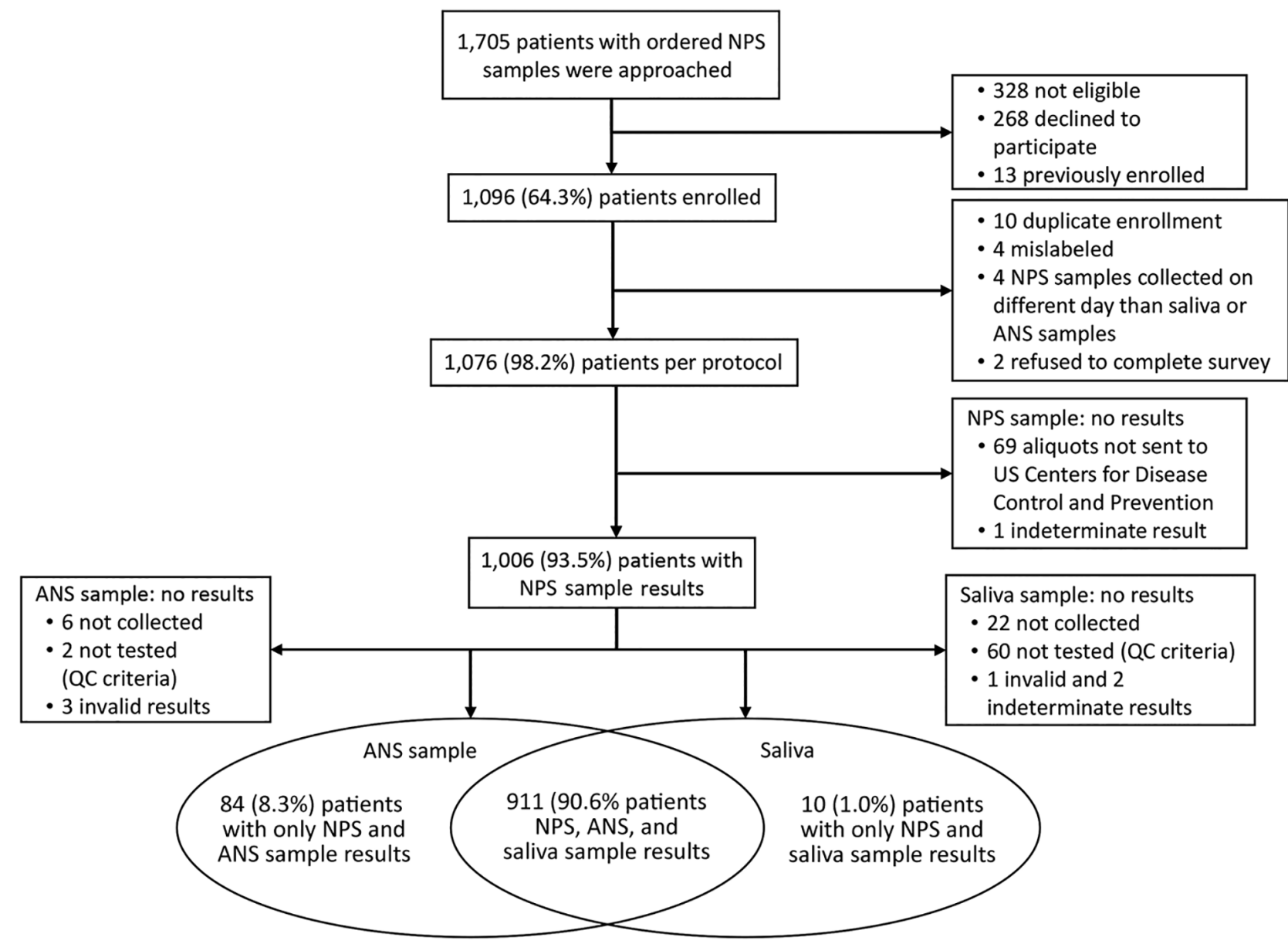

Figure 1. Flowchart of patient enrollment and sample results for investigation of the effects of patient characteristics on self-collected and healthcare worker-collected samples for severe acute respiratory syndrome coronavirus 2 testing, Atlanta, Georgia, USA. ANS, anterior nasal swab; NPS, nasopharyngeal swab; QC, quality control. 
Most $(93.5 \% ; 1,006)$ participants provided an NPS sample, of which $8.0 \%(80 / 1,006)$ tested positive for SARS-CoV-2. A total of 911 participants had an RTPCR result for all 3 specimens (i.e., saliva, ANS, and NPS samples), 10 participants had results for only saliva and NPS samples, and 84 participants had results for only ANS and NPS samples (Figure 1).

\section{Performance of Self-Collected Sample Types}

Among 995 participants who provided ANS and NPS samples that produced definitive results, 963 $(96.8 \%)$ had concordant results $(\mathrm{K}=0.73,95 \% \mathrm{CI}$ 0.64-0.82). Compared with NPS samples, ANS samples had 59\% sensitivity (95\% CI 47\%-70\%), 100\% specificity (95\% CI 100\%-100\%), 100\% PPV (95\% CI 92\%-100\%), and 97\% NPV (95\% CI 95\%-98\%). Among 921 participants who provided saliva and NPS samples that produced definitive results, 894 $(97.1 \%)$ had concordant results $(\mathrm{K}=0.76,95 \% \mathrm{CI}$ 0.67-0.85). Compared with NPS samples, saliva had $68 \%$ sensitivity (95\% CI 55\%-78\%), 99\% specificity (95\% CI 99\%-100\%), 90\% PPV (95\% CI 79\%-97\%), and $97 \%$ NPV (95\% CI 96\%-98\%).

To understand the benefit of using both selfcollected specimens for diagnosis, we analyzed data from 1,005 participants who had definitive results for $>1$ self-collected specimen. We found that 977 (97.2\%) had concordant results between the selfcollected combination and NPS samples $(\kappa=0.79$, 95\% CI 0.71-0.86). Using NPS as the comparator, we found self-collected combination samples had 71\% sensitivity (95\% CI 60\%-81\%), 99\% specificity (95\% CI 99\%-100\%), 92\% PPV (95\% CI 82\%-97\%), and 98\% NPV (95\% CI 96\%-98\%). When any positive was used as the comparator, we observed little change in the overall findings: the overall sensitivity of the ANS swab sample decreased slightly, the sensitivity of saliva samples increased slightly, and sensitivity of self-collected combination samples increased slightly (Appendix Table 1).

\section{Sensitivity by Patient Characteristics and Symptoms}

Saliva and self-collected combination samples had higher overall sensitivities than ANS samples; this pattern was reflected among men, participants 18-29 years of age and 50-59 years of age, and Black (nonHispanic) participants (Table 2). Among Hispanic/ Latinx participants, sensitivity was significantly lower for saliva and self-collected combination samples. Sensitivity was higher among those not reporting any chronic medical conditions, whose reason for hospital visit was a COVID-19-related concern or whose chief complaint included COVID-19-like symptoms, who reported close contact to a COVID-19 patient during the previous $\leq 14$ days, and who did not report a previous positive COVID-19 test. Sensitivity was lower among participants who reported a previous positive COVID-19 test (Table 2).

Sensitivity was higher among samples from currently symptomatic participants (62\% for ANS, $72 \%$ for saliva, and $76 \%$ for self-collected combination) and was highest among samples from participants who provided samples 3-7 days after symptom onset (80\% for ANS, $85 \%$ for saliva, and $88 \%$ for self-collected combination); these differences were statistically significant for ANS and self-collected combination samples $(p<0.05)$. Sensitivity was higher for most individual symptoms, but highest among participants reporting measured fever, congestion or runny nose, new loss of smell, new loss of taste, cough, or subjective fever. Similarly, sensitivity was higher among participants who met most symptom case definitions, but highest among patients who had influenza-like illness, COVID-19-like symptoms, or upper respiratory symptoms accompanied by loss of smell or taste. Sensitivity was lower among patients with nonconstitutional symptoms (Table 2).

\section{$\mathrm{C}_{\mathrm{t}}$ Values}

Among 46 participants with positive ANS and NPS samples, 85\% (for PCR target N1) and 78\% (for PCR target N2) had an ANS sample with a higher $C_{t}$ value than that of its paired NPS sample (Figure 2, panels A, B). We observed a moderate positive correlation between the $C_{t}$ values of ANS and NPS samples $(\mathrm{r}=0.75$ for $\mathrm{N} 1, \mathrm{r}=0.71$ for $\mathrm{N} 2)$; both targets had median NPS $C_{t}$ values of 22.8 (range 14.6-34.1 for N1, 14.7-35.0 for N2). Among 46 participants with positive saliva and NPS samples, 57\% (N1) and 59\% (N2) had a saliva sample with a higher $C_{t}$ value than that of its paired NPS sample (Figure 2, panels C, D). We observed a low positive correlation between the $C_{t}$ values of saliva and NPS samples $(r=0.53$ for both $\mathrm{N} 1$ and N2); targets had median NPS $\mathrm{C}_{\mathrm{t}}$ values of 23.1 (range 14.6-38.3) for N1 and 23.8 (range 14.7-37.7) for $\mathrm{N} 2$. When limiting the analysis to the 72 participants who had 3 definitive and $\geq 1$ positive result, $C_{t}$ values were lowest when all paired specimens were SARSCoV-2-positive; for N1, the median $C_{t}$ values were 27.2 for ANS, 24.9 for saliva, and 22.6 for NPS samples (Figure 3). When $\leq 2$ specimens were positive, all specimens had median $C_{t}$ values $>30$. Participants who did not have COVID-19 symptoms had higher median NPS $C_{t}$ values (33.5 for N1, 34.4 for N2) than did those who reported $\geq 1$ COVID-19 symptom (25.6 for $\mathrm{N} 1, \mathrm{p}=0.03 ; 26.9$ for $\mathrm{N} 2, \mathrm{p}=0.03$ ). Among those 


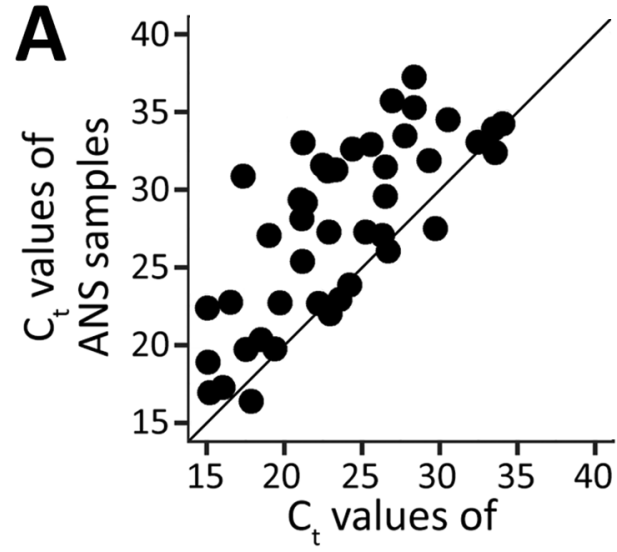

NPS samples

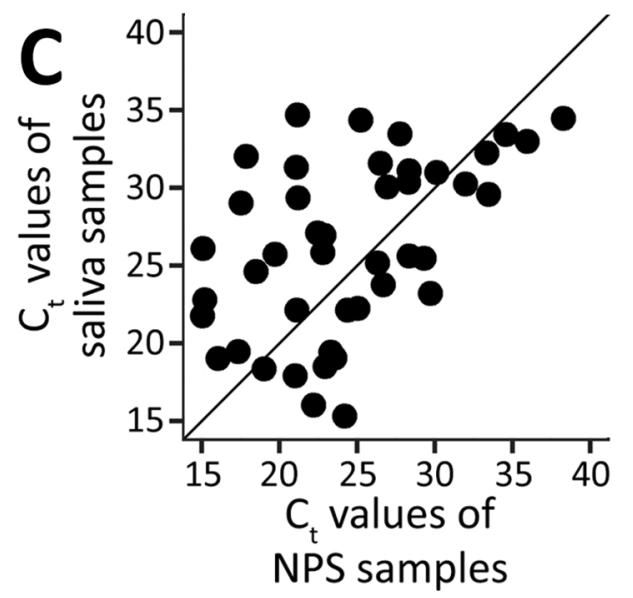

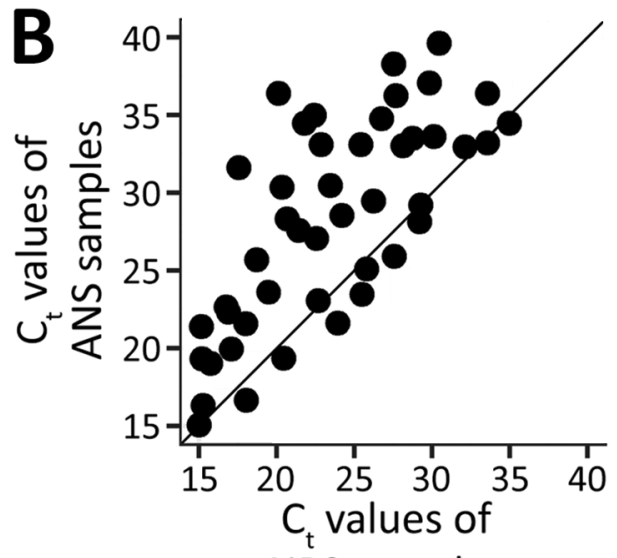

NPS samples

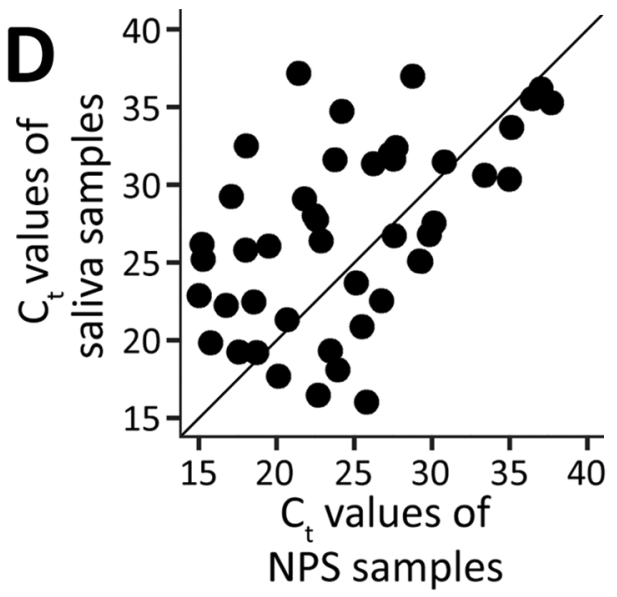

Figure 2. $C_{t}$ values of selfcollected and healthcare workercollected samples for severe acute respiratory syndrome coronavirus 2 testing, Atlanta, Georgia, USA. PCR completed using CDC 2019-nCoV RealTime Reverse Transcriptase PCR Diagnostic Panel (15). A) ANS and NPS samples at PCR target N1. B) ANS and NPS samples at PCR target N2. C) NPS and saliva samples at PCR target N1. D) NPS and saliva samples at PCR target N2. ANS, anterior nasal swab; $C_{t}$, cycle threshold; NPS, nasopharyngeal swab. reporting COVID-19 symptoms, participants who had symptom onset $\leq 1$ week before testing had the lowest median NPS $C_{t}$ values (23.5 vs. 30.8 for N1, $\mathrm{p}<0.01 ; 24.2$ vs. 33.3 for $\mathrm{N} 2, \mathrm{p}<0.01)$.

\section{Discussion}

In this investigation, we found that self-collected saliva samples had a higher sensitivity than self-collected ANS samples (68\% vs. 59\%) compared with HCWcollected NPS samples. However, each sample type had lower sensitivity than suggested by most previously published data $(3,6,19-21)$. The self-collected combination had a higher sensitivity $(71 \%)$ than NPS samples. We found that the sensitivity of self-collected samples (separately and in combination) differed according to patient characteristics. The presence of COVID-19 symptoms at time of specimen collection and the time since symptom onset affected sensitivity. We also noted differences in sensitivity across demographic groups, possibly reflecting differences in access to care or care-seeking behavior rather than differences in viral shedding. Our results illustrate that certain patient characteristics are associated with the sensitivity of self-collected specimens used for RT-PCR.

We found lower sensitivities for saliva and ANS samples than those for most other published studies, including 2 recent meta-analyses that found saliva samples to have sensitivities of $83.2 \%$ and $86.9 \%$ $(21,22)$. Many studies showing high sensitivity of selfcollected specimens enrolled symptomatic patients who were recently hospitalized for confirmed COVID-19 or whose symptom onset was $\leq 1$ week before sample collection $(3,4)$. A strength of our investigation was that we included symptomatic and asymptomatic patients being tested for SARS-CoV-2 for screening and diagnostic purposes. Because a substantial proportion of patients infected with SARS-CoV-2 have asymptomatic or mild illness, physicians must be able to analyze results in the context of test sensitivity in patients with few or no symptoms. Sample sensitivity was highest among participants reporting symptom onset 3-7 days before sample collection. Similarly, another study found that the sensitivity of saliva samples was highest (95\%) among symptomatic patients tested $\leq 1$ week after symptom onset and lowest 
(50\%) among patients tested $>1$ week after symptom onset (23). The sample sensitivity differences among patients with different demographic characteristics might reflect differences in access to care or healthcare-seeking behavior. Delayed access to care might postpone specimen collection, decreasing the sensitivity of the samples. For example, Hispanic/Latinx participants who had a positive NPS sample had longer symptom duration compared with participants of other race/ethnicity categories (data not shown).

Using 2 self-collected specimens could increase overall test sensitivity, which reached $88 \%$ among participants whose symptoms began 3-7 days before sample collection. Similarly, Tan et al. (24) found that combining self-collected oropharynx and midturbinate swab and saliva results increased test sensitivity. Using multiple noninvasive specimens might improve SARS-CoV-2 detection in persons tested $\leq 1$ week after symptom onset and reduce demand for PPE and HCW exposure. However, testing multiple specimens might put additional strain on laboratory systems that are already overburdened. Pooling self-collected specimens before testing might alleviate some of this additional strain on laboratories, but this practice should be investigated further for accuracy.
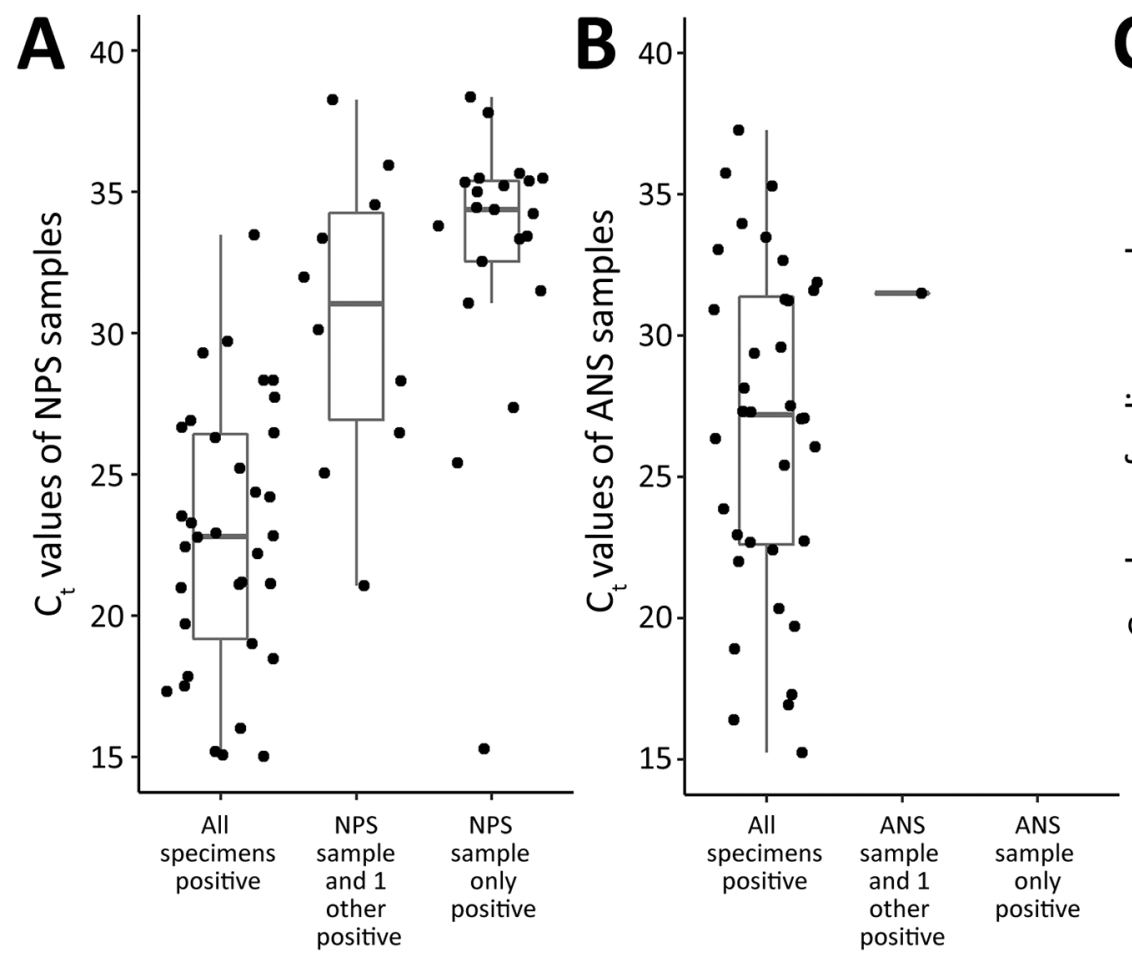

Similar to other studies, we found that most NPS samples had lower $C_{t}$ values than did their paired saliva and ANS samples $(4,25,26)$. We also found that median $C_{t}$ values were lowest when all 3 specimens were SARS-CoV-2-positive; the median $\mathrm{C}_{t}$ value increased to $>30$ when $\leq 2$ specimens were positive (Figure 3 ). The lower sensitivity in this investigation might be due to high $C_{t}$ value discordant specimens, which can occur as infection subsides. We also found slightly higher overall median $C_{t}$ values for NPS samples than reported in similar studies $(5,27)$. However, many of these previous studies were implemented earlier in the pandemic when previous infection or exposure was less common. Our investigation began after the first 2 peaks in Atlanta; by the end of enrollment, Atlanta was entering its third peak. When the $C_{t}$ value of the NPS sample is high, discordance with the self-collected specimens also could increase. Salvatore et al. (28) found that $C_{t}$ values for NPS samples were lowest $\leq 1$ week after symptom onset. Furthermore, Wolfel et al. (29) found viral subgenomic mRNA in throat swab specimens collected $<5$ days after symptom onset and in sputum samples taken 4-11 days after symptom onset, indicating active infection. Although $C_{t}$ values are not directly correlated with viral load, they provide a semiquantitative assessment of viral RNA concentration.

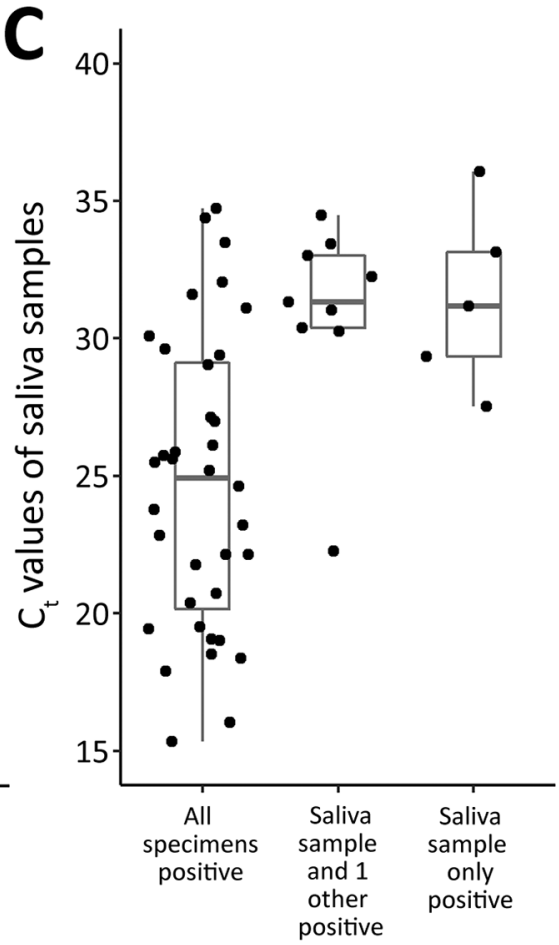

Figure 3. $C_{t}$ values of self-collected and healthcare worker-collected samples for $\mathrm{N} 1$ target of severe acute respiratory syndrome coronavirus 2 PCR, Atlanta, Georgia, USA. PCR completed using CDC 2019-nCoV Real-Time Reverse Transcriptase PCR Diagnostic Panel (15). Horizontal lines within boxes indicate medians; box tops and bottoms indicate 25th and 75th percentiles; whiskers indicate the range. ANS, anterior nasal swab; $\mathrm{C}_{\mathrm{t}}$, cycle threshold; NPS, nasopharyngeal swab. 
Specimen collection method might also affect sensitivity. Procop et al. (5) compared NPS and enhanced saliva samples (i.e., self-collected nasal secretions or mucus, phlegm, and saliva stored in a single tube) and found $100 \%$ positive agreement and $99.4 \%$ negative agreement. This method of saliva collection provides a mixture of upper and lower respiratory secretions, thereby enabling detection for a longer time after symptom onset. We used general spitting for saliva collection, which has the lowest sensitivity estimate in comparison with other saliva collection methods (22). General spitting does not require special devices or transport media, enabling our methods and results to be broadly applicable. The duration and pressure applied while swabbing the anterior nares also might affect ANS sample quality. The sequence of specimen collection, which was not always clear from published studies, could also affect sensitivity (19). In our investigation, saliva and ANS samples were collected before NPS samples. Collecting ANS samples after NPS samples could displace virus from nasopharyngeal tissue or midturbinate before the swab leaves the nares, thereby biasing self-collected ANS toward higher sensitivity.

The first limitation of our study is that because we used a cross-sectional design, we did not have information on whether patients were asymptomatic or presymptomatic at specimen collection; we also did not have data on disease severity. In presymptomatic patients, samples might have been collected too early to detect viral RNA in some or all specimen types. Second, we lacked the statistical power to detect significant differences in sensitivity by most variables because the initial sample size calculation assumed self-collected specimens to have $\geq 90 \%$ sensitivity. Third, all responses were self-reported and could have been affected by recall bias. Fourth, the CDC 2019-nCoV rRT-PCR Diagnostic Panel has a higher limit of detection than many commercially available, high-throughput assays (30), limiting our ability to detect lower concentrations of viral RNA. However, the clinical and public health utility of detecting these lower concentrations is unknown. Fifth, the CDC 2019-nCoV rRT-PCR Diagnostic Panel does not currently include saliva; instead, the assay was designed for qualitative detection of nucleic acid from SARS-CoV-2. Of 1,006 NPS samples, 64 aliquots did not meet storage requirements approved under the assay's instructions for use (samples should be stored at $4^{\circ} \mathrm{C}$ for $\leq 72$ hours after collection) (15). Because some samples were stored for longer than recommended, viral RNA degradation might have affected the assay's performance. Because the CDC RT-PCR results were not used for clinical care, excluding these specimens did not change sensitivity; furthermore, CDC RT-PCR results were in concordance with the hospital's RT-PCR results (data not shown). As a result, we decided to include these specimens in the analysis (Appendix). Our findings might not be generalizable to other assays or techniques. Sixth, heterogenous selfcollection coaching techniques might have introduced differences in the quality of samples collected under the guidance of different interviewers. Finally, PCR does not indicate whether active replication is occurring. Therefore, we are unable to determine whether patients with positive NPS samples but negative saliva or ANS samples have older infections or if the self-collected specimens are less sensitive than NPS samples. Additional laboratory testing is required to clarify the viability of different specimen types and how viability affects clinical presentation and transmissibility. Our study highlights that the sensitivities of saliva and ANS samples are different than that of NPS samples. These findings show that physicians should consider the patient's clinical history, exposures, and time of symptom onset when interpreting PCR results.

Overall, the sensitivities of ANS and saliva samples were lower than that of NPS samples from patients being tested for SARS-CoV-2 for diagnostic and screening purposes. The sample sensitivity was highest among participants with symptom onset within 3-7 days of specimen collection, especially when the reason for the patient visit was COVID-19related, and those not reporting a previous positive test. Encouraging persons to seek testing within a week of symptom onset could increase the accuracy and usefulness of self-collected specimens used for diagnosing SARS-CoV-2 infections. It is important that clinicians are aware of how differences in patient characteristics and specimen type can affect test sensitivity. Testing programs and clinical settings might consider patient characteristics, previous test results, and timing of symptom onset when determining which specimen types to use.

Members of the CDC COVID-19 Response Team: Mila Cohen, Amadea Britton, Courtney T. Callahan, Jamila Fonseka, Elfriede Agyemang, Miriam J. Lawson, Molly Deutsch-Feldman, Tejpratap S. P. Tiwari, Samira Sami, Hong Tao, Tiffany M. Aholou, Katherine M. Butler, D. Joseph Sexton, Nicolas Wiese, Raydel Anderson, Kay W. Radford, Gimin Kim, Jennifer M. Folster, Magdalena Medrzycki, Patricia L. Shewmaker, Baoming Jiang, Jan Vinje, Rashi Gautam, Slavica Mijatovic-Rustempasic, Leslie Barclay, Michelle Adamczyk, Erin Breaker, Davina 
Campbell, Lori M. Spicer, Carrie A. Sanders, Lisa Tran, William A. Furin, Amanda Lyons, Hollis Houston, Karlos Crayton, J. Carrie Whitworth, Natashia R. Reese, David R. Lonsway, Rocio Balbuena, K. Allison Perry-Dow, Monica Y. Chan, Alison Laufer Halpin, and Wendi Kuhnert-Tallman.

\section{Acknowledgments}

We thank Francisco Averhoff, Luis Lowe, Brandi Limbago, and Susan Ray for initial project conception and Tiffany Li for sample size calculation. We thank the CDC COVID-19 Response Epidemiology Task Force field team logistics coordinators (Gerardo Garcia-Lerma, Lauren Franco, Anupama Shankar, Adam Wharton) who helped with everything from staffing to procuring supplies and Laura Hughes-Baker for her coordination with the CDC laboratories. We want to thank the CDC COVID-19 Surge Laboratory for specimen extraction and SARS-CoV-2 rRT-PCR testing (Gillian A. McAllister, Kamile Rasheed, Uzma A. Ansari, Amelia Bhatnagar, Maria Karlsson, M. Shannon Keckler, Katherine Butler, D. Joseph Sexton, Dexter Thompson, Congrong Miao, Min-hsin Chen, Brent M. Jenkins, Nhien Tran, Srinivasan Velusamy, Lalitha Gade, Kashif Sahibzada, Renee Galloway, Phili Wong, HaoQiang Zheng, Amy Hopkins, Eric Katz, Hannah Browne, Kenny Nguyen, Mathew Esona, Sung-Sil Moon, Theresa Bessey, Preeti Chhabra, and Leeann Smart). Finally, we thank the healthcare workers at Grady Memorial Hospital for their dedication and assistance and the participants for their participation despite the circumstances that brought them to the hospital.

Emory University received funding from the CDC Foundation for this work.

\section{About the Author}

Ms. Smith-Jeffcoat is an epidemiologist with the Respiratory Viruses Branch, Division of Viral Diseases, National Center for Immunization and Respiratory Diseases, Centers for Disease Control and Prevention, Atlanta, Georgia, USA. Her research interests include respiratory disease epidemiology and global tuberculosis epidemiology, especially drug-resistant tuberculosis.

\section{References}

1. World Health Organization. COVID-19 weekly epidemiological update-27 January 2021. 2021 Jan 27 [cited 2021 Feb 9]. https:/ / www.who.int/publications/m/item/ weekly-epidemiological-update---27-january-2021

2. Centers for Disease Control and Prevention. Interim guidelines for collecting, handling, and testing clinical specimens for COVID-19. 2021 [cited 2021 Jan 29]. https:/ / www.cdc.gov/ coronavirus/2019-ncov/lab/guidelines-clinical-specimens.html
3. Tu Y-P, Jennings R, Hart B, Cangelosi GA, Wood RC, Wehber K, et al. Swabs collected by patients or health care workers for SARS-CoV-2 testing. N Engl J Med. 2020;383:494-6. https://doi.org/10.1056/NEJMc2016321

4. McCormick-Baw C, Morgan K, Gaffney D, Cazares Y, Jaworski K, Byrd A, et al. Saliva as an alternate specimen source for detection of SARS-CoV-2 in symptomatic patients using Cepheid Xpert Xpress SARS-CoV-2. J Clin Microbiol. 2020;58:e01109-20. https:// doi.org/10.1128/JCM.01109-20

5. Procop GW, Shrestha NK, Vogel S, Van Sickle K, Harrington S, Rhoads DD, et al. A direct comparison of enhanced saliva to nasopharyngeal swab for the detection of SARS-CoV-2 in symptomatic patients. J Clin Microbiol. 2020;58:e01946-20. https:// doi.org/10.1128/JCM.01946-20

6. Hanson KE, Barker AP, Hillyard DR, Gilmore N, Barrett JW, Orlandi RR, et al. Self-collected anterior nasal and saliva specimens versus health care worker-collected nasopharyngeal swabs for the molecular detection of SARS-CoV-2. J Clin Microbiol. 2020;58:e01824-20. https://doi.org/10.1128/JCM.01824-20

7. Payne DC, Smith-Jeffcoat SE, Nowak G, Chukwuma U, Geibe JR, Hawkins RJ, et al.; CDC COVID-19 Surge Laboratory Group. SARS-CoV-2 infections and serologic responses from a sample of U.S. Navy service members USS Theodore Roosevelt, April 2020. MMWR Morb Mortal Wkly Rep. 2020;69:714-21. https://doi.org/10.15585/ mmwr.mm6923e4

8. Yousaf AR, Duca LM, Chu V, Reses HE, Fajans M, Rabold EM, et al. A prospective cohort study in nonhospitalized household contacts with severe acute respiratory syndrome coronavirus 2 infection: symptom profiles and symptom change over time. Clin Infect Dis. 2020 Jul 28 [Epub ahead of print]. https:// doi.org/10.1093/cid/ ciaa1072

9. Buitrago-Garcia D, Egli-Gany D, Counotte MJ, Hossmann S, Imeri $\mathrm{H}$, Ipekci AM, et al. Occurrence and transmission potential of asymptomatic and presymptomatic SARS-CoV-2 infections: a living systematic review and meta-analysis. PLoS Med. 2020;17:e1003346. https:/ / doi.org/ 10.1371/journal.pmed.1003346

10. Sun J, Xiao J, Sun R, Tang X, Liang C, Lin H, et al. Prolonged persistence of SARS-CoV-2 RNA in body fluids. Emerg Infect Dis. 2020;26:1834-8. PubMed https://doi.org/10.3201/ eid2608.201097

11. COVID-19 Investigation Team. Clinical and virologic characteristics of the first 12 patients with coronavirus disease 2019 (COVID-19) in the United States. Nat Med. 2020;26:861-8. PubMed https:/ / doi.org/10.1038/ s41591-020-0877-5

12. Grady Health System. Grady fast facts. 2018 [cited 2021 Jan 29]. https:/ / www.gradyhealth.org/wp-content/ uploads/2018/07/Grady_Fast_Facts.pdf

13. Centers for Disease Control and Prevention. How to collect your anterior nasal swab sample for COVID-19 testing. 2020 [cited 2021 Feb 12]. https:/ / www.cdc.gov/ coronavirus/2019-ncov/testing/How-To-Collect-AnteriorNasal-Specimen-for-COVID-19.pdf

14. Lu X, Wang L, Sakthivel SK, Whitaker B, Murray J, Kamili S, et al. US CDC real-time reverse transcription PCR panel for detection of severe acute respiratory syndrome coronavirus 2. Emerg Infect Dis. 2020;26:1654-65. https://doi.org/10.3201/eid2608.201246

15. United States Food and Drug Administration. Emergency use authorization: CDC 2019-nCoV Real-Time RT-PCR Diagnostic Panel. 2020 [cited 2021 Mar 2]. https:/ / www.fda. gov/media/134919/download 
16. Boehmer TK, DeVies J, Caruso E, van Santen KL, Tang S, Black CL, et al. Changing age distribution of the COVID-19 pandemic - United States, May-August 2020. MMWR Morb Mortal Wkly Rep. 2020;69:1404-9. https:/ / doi.org/10.15585/ mmwr.mm6939e1

17. Council of State and Territorial Epidemiologists. Update to the standardized surveillance case definition and national notification for 2019 novel coronavirus disease (COVID-19). 2020 [cited 2021 Jan 29]. https://cdn.ymaws.com/www.cste. org/resource/resmgr/ps/positionstatement2020/ Interim-20-ID-02_COVID-19.pdf

18. World Health Organization. RSV surveillance case definitions. 2020 [cited 2021 January 29]; https:/ / www. who.int/influenza/rsv/rsv_case_definition/en

19. Altamirano J, Govindarajan P, Blomkalns AL, Kushner LE, Stevens BA, Pinsky BA, et al. Assessment of sensitivity and specificity of patient-collected lower nasal specimens for severe acute respiratory syndrome coronavirus 2 testing [Erratum in: JAMA Netw Open. 2020;3: e2014910]. JAMA Netw Open. 2020;3:e2012005. https:// doi.org/10.1001/ jamanetworkopen.2020.12005

20. Kojima N, Turner F, Slepnev V, Bacelar A, Deming L, Kodeboyina S, et al. Self-collected oral fluid and nasal swabs demonstrate comparable sensitivity to clinician-collected nasopharyngeal swabs for the detection of SARS-CoV-2. Clin Infect Dis. 2020 Oct 19 [Epub ahead of print]. https://doi.org/10.1093/cid/ciaa1589

21. Butler-Laporte G, Lawandi A, Schiller I, Yao M, Dendukuri N, McDonald EG, et al. Comparison of saliva and nasopharyngeal swab nucleic acid amplification testing for detection of SARS-CoV-2: a systematic review and meta-analysis. JAMA Intern Med. 2021;181:353-60. https://doi.org/10.1001/jamainternmed.2020.8876

22. Bastos ML, Perlman-Arrow S, Menzies D, Campbell JR. The sensitivity and costs of testing for SARS-CoV-2 infection with saliva versus nasopharyngeal swabs: a systematic review and meta-analysis. Ann Intern Med. 2021;174:501-10. https:/ / doi.org/10.7326/M20-6569

23. Migueres M, Mengelle C, Dimeglio C, Didier A, Alvarez M, Delobel $\mathrm{P}$, et al. Saliva sampling for diagnosing SARS-CoV-2 infections in symptomatic patients and asymptomatic carriers.
J Clin Virol. 2020;130:104580. https:/ / doi.org/10.1016/ j.jcv.2020.104580

24. Tan SY, Tey HL, Lim ETH, Toh ST, Chan YH, Tan PT, et al. The accuracy of healthcare worker versus self collected (2-in-1) oropharyngeal and bilateral mid-turbinate (OPMT) swabs and saliva samples for SARS-CoV-2. PLoS One. 2020;15:e0244417. https:// doi.org/10.1371/journal.pone.0244417

25. Landry ML, Criscuolo J, Peaper DR. Challenges in use of saliva for detection of SARS CoV-2 RNA in symptomatic outpatients. J Clin Virol. 2020;130:104567. https:/ / doi.org/ 10.1016/j.jcv.2020.104567

26. Van Vinh Chau N, Lam VT, Dung NT, Yen LM, Minh NNQ, Hung LM, et al.; Oxford University Clinical Research Unit COVID-19 Research Group. The natural history and transmission potential of asymptomatic severe acute respiratory syndrome coronavirus 2 infection. Clin Infect Dis. 2020;71:2679-87. https://doi.org/10.1093/cid/ciaa711

27. Williams E, Bond K, Zhang B, Putland M, Williamson DA. Saliva as a noninvasive specimen for detection of SARS-CoV-2. J Clin Microbiol. 2020;58:e00776-20. https://doi.org/10.1128/JCM.00776-20

28. Salvatore PP, Dawson P, Wadhwa A, Rabold EM, Buono S, Dietrich EA, et al. Epidemiological correlates of polymerase chain reaction cycle threshold values in the detection of SARS-CoV-2. Clin Infect Dis. 2020;72:e761-7. https://doi.org/10.1093/cid/ciaa1469

29. Wölfel R, Corman VM, Guggemos W, Seilmaier M, Zange S, Müller MA, et al. Virological assessment of hospitalized patients with COVID-2019 [Erratum in: Nature. 2020; 588:E35]. Nature. 2020;581:465-9. https:/ / doi.org/10.1038/ s41586-020-2196-x

30. Food and Drug Administration. Individual EUAs for molecular diagnostic tests for SARS-CoV-2. 2021 [cited 2021 Feb 2]. https:/ / www.fda.gov/medical-devices/coronavirusdisease-2019-covid-19-emergency-use-authorizationsmedical-devices/vitro-diagnostics-euas\#individual-molecular

Address for correspondence: Sarah E. Smith-Jeffcoat, Centers for Disease Control and Prevention, 1600 Clifton Road NE, Mailstop H24-5, Atlanta, GA 30329-4027, USA; email: uyi7@cdc.gov 